\title{
EL USO DE DROGAS EN EL PERSONAL DE ENFERMERÍA
}

\section{DRUG USE IN NURSING WORKERS}

\author{
LOURDes Díaz C. * \\ Célia Marina Ulloa ** \\ Fabiana Taubert de Freitas ${ }^{* * *}$ \\ LiLiana AMORIM A. ${ }^{* * * *}$ \\ Maria Célia Barcelos D. ${ }^{* * * *}$ \\ Sandra Verónica Valenzuela S. \\ Maria Lúcia do Carmo CruZ R. ${ }^{* * * * * *}$
}

\begin{abstract}
RESUMEN
El abuso de drogas produce sufrimiento orgánico y psíquico a la persona y alteraciones multidimensionales que son reflejadas hacia la sociedad, familia, los estudios y el trabajo. Este artículo presenta un análisis respecto a la utilización de drogas en el personal de enfermería y la relación entre uso y abuso de estas sustancias y el mundo del trabajo de enfermería, con el objetivo de formular observaciones sobre la enfermera como una persona que podría usar drogas ante los problemas laborales que enfrenta. Se hacen consideraciones sobre el trabajo del personal de enfermería y los problemas ocupacionales, buscando comprender mejor la relación entre las drogas y los usuarios. Propuestas para minimizar el problema, así como, la necesidad de una mayor atención a la salud y educación permanente en materia de drogas, destinadas a ampliar la capacidad de hacer frente a este problema en los mismos trabajadores.
\end{abstract}

Palabras clave: Trabajadores, enfermería, uso indebido de drogas.

\begin{abstract}
Drug abuse produces organic and mental suffering to the individual and multidimensional changes that are reflected back to society, family, school and work. This paper presents an analysis on drug use in nursing and the relationship between use and abuse of these substances and the world of nursing work, in order to comment on the nurse as a person who might use drugs facing labor problems. Considerations are made on the work of nurses and occupational problems, then we try to understand better the relationship between drugs and users and finally, generating suggestions for minimizing the problem. Provides suggestions to minimize the problem and highlights the need for greater attention to health and continuing education on drugs, designed to expand capacity to cope with this problem in the same workers.
\end{abstract}

Key words: Workers, nursing, drug abuse.

Fecha recepción: 31.05.10 Fecha aceptación: 03.05.11

\footnotetext{
*Enfermera del Programa Nacional de la Secretaría de Salud de Honduras. E-mail: lourdesdiazcanaca@yahoo.es

${ }^{*}$ Enfermera del Programa Nacional de la Secretaría de Salud de Honduras. E-mail: celiamarina_7@hotmail.com

${ }^{* * *}$ Doctorandas del Programa de Enfermería Fundamental de la Escola de Enfermagem de Ribeirão Preto, Universidade São Paulo, Brazil. E -mail: ftaubert@eerp.usp.br

${ }^{* * * *}$ Doctorandas del Programa de Enfermería Fundamental de la Escola de Enfermagem de Ribeirão Preto, Universidade São Paulo, Brazil. E -mail: liliana@eerp.usp.br

${ }^{* * * * *}$ Enfermera. Profesora Doctora de la Escola de Enfermagem de Ribeirão Preto, Universidade São Paulo, Brazil. E-mail: macdalri@eerp.usp.br

${ }^{* * * * * *}$ Doctora en Enfermería. Docente Depto. Enfermería, Universidad de Concepción, Chile. E-mail: svalenzu@udec.cl

${ }^{* * * * * *}$ Enfermera. Profesor de la Escola de Enfermagem de Ribeirão Preto, USP. Departamento de Enfermagem Geral e Especializada. Ribeirão Preto - São Paulo - Brasil. E-mail: avrmlccr@eerp.usp.br
} 


\section{INTRODUCCIÓN}

El abuso de drogas o farmacodependencia implica una autoadministración de compuestos o sustancias farmacodependientes psicoactivas. Este consumo produce sufrimiento orgánico y psíquico a la persona y alteraciones multidimensionales que son reflejadas hacia la sociedad, familia, los estudios y el trabajo (1).

La temática del abuso de drogas se ha considerado importante y merece la preocupación de las organizaciones mundiales, gubernamentales y no gubernamentales (1) y la sociedad en su conjunto (2-4). En 2002, el 8,9\% de la causa general de las enfermedades fueron el resultado de este consumo, en el 2000, el tabaco representaba el 4,1\%, 4 el alcohol y drogas ilícitas el 0,8\%. Gran parte de la relación establecida a dicho uso y farmacodependencia se generó desde la salud y de los problemas sociales que ocasionaban, incluidas las enfermedades causadas por el consumo de drogas inyectables (2).

Las sustancias que generan farmacodependencia o psicoactivas se han convertido en un problema multidimensional, no circunscrito a una simple relación entre la persona y la droga. Es importante considerar la interacción que ocurre entre ellos en un determinado contexto, prestando atención a los valores y creencias, sus características sociales, económicas y políticas, incluido el trabajo (3).

Desde esta perspectiva no se debe ignorar la dimensión humana del trabajo, tanto en términos de las consecuencias del uso y abuso, que puede afectar las relaciones y acciones, como las acciones propias de los factores de organización y desarrollo del trabajo, como factores capaces de intervenir en el complejo examen de la farmacodependencia (1).

Profesiones en riesgo para este abuso son aquellas que se desarrollan en ambientes estresantes, con trabajos nocturnos y que requieren de una atención constante (4) y este uso es cada vez más frecuente en el mundo del trabajo, alterando las relaciones laborales, interpersonales, familiares y la salud (4).

Contextos perjudiciales pueden favorecer la aparición de factores de riesgo para la ocurrencia de este fenómeno entre los profesionales de la salud por la oportunidad de acceso a los mismos $(3,4)$, extendiéndose, entre ellos, a los trabajadores de enfermería (5), que generalmente actúan en ambientes ocupacionales estresantes (6). Los efectos sobre la productividad van a ser expresados por los atrasos, el ausentismo, el aumento de ocurrencia de accidentes, las acciones inadecuadas y los conflictos interpersonales (1).

El trabajo de enfermería, desde sus diversas dimensiones y complejidades que significan el cuidado de la salud comprende situaciones relacionadas con la capacidad de los trabajadores para actuar frente al sufrimiento, dolor, enfermedad, muerte, y la exposición a variados factores de riesgo propiciados por el contacto con sustancias químicas, radiaciones, contaminantes biológicos, calor excesivo, sistema de turnos, horas extras, la rotación de personal, cuestiones relacionadas con los servicios de dirección de personal y gestión, entre otros $(3,5)$.

El equipo de enfermería posee una formación heterogénea, se encuentra conformado por trabajadoras/es técnicos y profesionales y realiza en común el cuidado de enfermería. Los clientes que reciben este cuidado tienen derecho a una atención de calidad, favorecida por trabajadores que desarrollan su quehacer en conformidad con buenas relaciones interpersonales, conocimientos éticos y legales, y con conocimientos y habilidades técnico-científicas. Para ofrecer este tipo de asistencia necesitan mantenerse sanos física y mentalmente, lo que se contrapone al uso y abuso de drogas psicotrópicas.

El abuso de sustancias entre los profesionales de la salud es un hecho y se ha convertido en una preocupación de las autoridades sanitarias de varios países, sin embargo, en Brasil esta discusión está en una fase preli- 
$\operatorname{minar}(5)$.

En este sentido, este texto reflexiona sobre la relación entre uso y abuso de estas sustancias en el mundo del trabajo de enfermería, con el objetivo de formular observaciones sobre la enfermera como una potencial consumidora de drogas. Así, se analiza el trabajo realizado y los problemas laborales que enfrenta y luego se busca determinar y entender mejor a los consumidores de drogas. Finalmente, se realizan consideraciones sobre la enfermería como una usuaria de estas sustancias y algunas sugerencias para minimizar este problema complejo y preocupante.

\section{EL TRABAJO Y SU IMPACTO SOBRE EL PERSONAL DE ENFERMERÍA}

En las instituciones de salud el equipo de enfermería tiene contacto directo con los pacientes, atendiendo sus demandas y las de sus familias y realiza actividades dependientes e interdependientes. Sin embargo, es común en los servicios de salud condiciones de trabajos inadecuados y ambientes adversos, con la presencia de factores de riesgo, biológicos, físicos, químicos y ergonómicos, entre otros (3, 7-9).

Las condiciones de trabajo implican una rutina estresante, elevado número de horas trabajadas, pacientes atendidos en situaciones complejas, turnos, bajos salarios y falta de reconocimiento del trabajo de este personal $(5,8,9)$. Este último aspecto redunda, incluso, en dificultades en el desempeño del trabajador, ya que muchas veces, se percibe desvalorizado y poco respetado (3). Al mismo tiempo que se idealiza su quehacer hay frustración por el escaso reconocimiento $\mathrm{y}$ desvalorización como trabajador (7). El poliempleo también es frecuente como en otros miembros del equipo de salud (10).

Ambientes peligrosos e insalubres favorecen condiciones adversas para la salud y la satisfacción personal del equipo de enferme- ría, generando exceso de actividad laboral física y mental y la inadecuación física de la relación de trabajo, factores determinantes para la ocurrencia de accidentes y enfermedades profesionales $(8,9)$.

Esta lista de factores que favorece la enfermedad, con riesgos de accidentes y dolencias de tipo física y de fuerte carga emocional, generan sufrimiento psicológico, dada la elevada presión social y psicológica que ocurre en el trabajo (7). Los factores de riesgo y condiciones de trabajo inadecuadas pueden causar o agravar los problemas de salud preexistentes $(8,9)$.

Los accidentes que ocurren en estos trabajadores son, entre otros, con objetos corto-punzantes $(7,11)$. Las enfermedades más frecuentes que los afectan son los trastornos musculo-esqueléticos (12) que lesionan la columna vertebral (13), trastornos del sistema respiratorio, problemas genitourinarios, enfermedades mal definidas, alteraciones en los órganos de los sentidos, enfermedades infecciosas (14), dolor en las extremidades inferiores, cambios de humor, trastornos del sueño y várices (7), la aparición de trastornos mentales debido al alto nivel de estrés laboral $(6,7)$ y otros problemas.

Para garantizar el cuidado continuado, el trabajo se realiza en horarios fijos y / o rotativos, que pueden afectar la salud de algunas trabajadoras y, particularmente, generar trastornos neuropsiquiátricos, cardiovasculares y gastrointestinales (15). El trabajo en sistema de turnos y/o de noche, la sobrecarga laboral cuantitativa y cualitativa, los problemas y las presiones diarias, responsabilidades excesivas, el trabajo lleno de rutinas, alteraciones en las relaciones interpersonales y la inestabilidad en el empleo pueden constituir factores de estrés laboral $(6,9)$ y generarles sufrimiento $(7,16)$. El ejercicio profesional puede interferir en la vida del trabajador, ocasionando cambios en las relaciones personales y familiares, limitando las actividades sociales y propiciando dificultades en la planificación de su vida como consecuencia 
del sistema de trabajo adoptado (15).

Diversas exigencias pueden crear condiciones favorecedoras para la búsqueda de un mundo inexistente de bienestar y tranquilidad que ofrece la droga $(3,4,17)$. Diversos factores facilitan el acercamiento al uso indebido de drogas, además de que muchas se encuentran disponibles en los ambientes ocupacionales $(3,4)$. Además de tener acceso a estos, los trabajadores son responsables por vigilar y controlar dichas sustancias (5); la facilidad para acceder a drogas puede considerarse un factor de riesgo y su uso está asociado con esta disponibilidad $(3,4)$.

Existe, entonces, la posibilidad real del abuso de sustancias psicoactivas (18), que se consumen con el fin de reducir los efectos del síndrome de desgaste profesional (17). El abuso de drogas puede ser entendido como una oportunidad que va a facilitar el manejo de su vida (5), pues estos compuestos pueden provocar sentimientos de placer, relajación, alivio del dolor y alteraciones en la percepción de la realidad $(3,4)$, promoviendo el olvido de los problemas cotidianos. Situaciones adversas, junto con la sobrecarga de trabajo motivan el uso de drogas o sustancias psicoactivas, conduciendo al trabajador de enfermería a una disminución del razonamiento, los reflejos y causar otros efectos nocivos (3).

A pesar de esto, el trabajo que realizan es distinguido del realizado por otros trabajadores de salud. Como es desarrollado a partir de una larga relación interpersonal con el paciente / familia / comunidad (3) también puede proporcionar satisfacción y bienestar al trabajador (19), sin los cuales sería difícil el ejercicio de su quehacer profesional (3).

\section{DROGAS Y USUARIOS}

La palabra droga se origina del persa droa (olor aromático), y rakab del hebreo (perfume) o del holandés antiguo droog (hoja seca); es considerada una forma química o mezcla que altera la función biológica y su estructura es capaz de modificar la función de los organismos vivos, dando lugar a cambios fisiológicos o de conducta (20). El uso de este término provoca muchas asociaciones, comprendiendo a sustancias capaces de alterar el estado de la conciencia y provocar dependencia en quienes las utilizan (4).

Las drogas ilícitas tienen su producción, venta y uso prohibido por ley; las sustancias lícitas son producidas y utilizadas legalmente, su comercialización es libre, a pesar de que pueden presentar perjuicio a la salud mental, física y social, lo que dependerá de múltiples factores tales como la cantidad, calidad y frecuencia de uso, entre otros (20).

Para la comprensión del concepto de "usuario", inicialmente se hace necesario definir el concepto de "no usuario", quien es aquella persona que no ha utilizado estas sustancias. A partir de aquí se entiende como usuario al consumidor leve, moderado, agudo y experimental/experimentador, clasificación basada en el consumo por día / semana / mes, a pesar de si es ocasional o esporádico, habitual, o funcional o dependiente y disfuncional, clasificación que relaciona el consumo con la ruptura o el mantenimiento de relaciones interpersonales (20).

Existen diversos factores que facilitan el consumo de drogas, incluyendo la proximidad de éstas con el usuario $(3,5)$.

\section{EL USO DE DROGAS EN ESTUDIANTES DE ENFERMERÍA}

Cuanto más es considerada lícita una sustancia química más se permite su uso y no se valora como ilegal. Las drogas resultan ser claves para la socialización, proporcionando y facilitando la complicidad y los procesos interactivos con los amigos. El estudiante ha heredado características de la academia y compone un grupo específico con sus pro- 
pios códigos funcionales (21).

Investigaciones muestran al trabajo de enfermería como factor de riesgo para el consumo de alcohol y otras drogas. Los estudiantes que asistieron a los dos últimos años de estudios de pregrado manifestaron quejas como estrés, irritabilidad y ansiedad, acompañadas de cansancio general, dolores de cabeza, tensión, cambios en los patrones de sueño y peso corporal. El trabajo en el período de internado fue señalado como de riesgo para el consumo de alcohol y drogas lícitas como el tabaco y ansiolíticos, e ilícitas, como la marihuana. Este consumo fue citado como una manera de reducir el desgaste o agotamiento psicofísico resultante de diversas actividades académicas, incluyendo el trabajo en los campos clínicos, también fue una forma de minimizar la ansiedad, los temores y preocupaciones que enfrentan en la vida cotidiana así como para relajarse y buscar placer (18).

Así también se ha estudiado el consumo de alcohol y drogas ilícitas entre los estudiantes de los cursos de primer año en medicina, biología y enfermería. La mayoría de los estudiantes de biología y de enfermería han informado utilizar, al menos una vez en la vida, el alcohol, el tabaco, la marihuana, los de medicina más alcohol, tabaco y los solventes. La juventud expresa su posible falta de madurez, lo que favorecería este uso (22).

Otra investigación informó la convivencia de estudiantes de enfermería con el uso y abuso de drogas psicoactivas en el ámbito universitario. Mostró que ellas conviven y también usan drogas psicoactivas por diversas razones, como el insomnio, el estrés, la obesidad, entre otros, y también en un intento de asemejarse a otros grupos que consumen alcohol y drogas en la universidad, al igual que otros estudios de estudiantes universitarios (23).

Una investigación con académicos de enfermería informó resultados de la utilización y actitudes relacionadas con el alcohol, tabaco, hojas de coca, tranquilizantes y anfetami- nas. Actitudes favorables relacionadas con las drogas se mostraron a través de la aprobación del uso de hojas de coca, alcohol, tranquilizantes y la desaprobación del consumo de cocaína. Se describió que el uso personal, en los últimos 12 meses, correspondió a uso de hojas de coca diariamente, alcohol menos de una vez por semana y tabaco de uso diario. Los estudiantes consumieron una primera vez motivados por la familia, amigos o novios (24). Se destaca que si los estudiantes de enfermería vivencian el uso de drogas para aliviar el estrés y reducir el desgaste psicofísico (18), podría ser ésta una situación que los motive a utilizarlas. Así, el consumo de drogas psicoactivas forma parte del contexto de los que trabajan en la enfermería y es importante tenerlo en cuenta (2).

\section{FUNCIONARIOS Y TRABAJADORES DE ENFERMERÍA}

El uso de drogas por parte del trabajador de enfermería representa la falta de cuidado de sí mismo; el cuidar traspasa el asistir y ayudar significa estar, que es una manera ética de ver, vivir y relacionarse con el mundo (17). Por otro lado, es difícil garantizar la competencia y la calidad del cuidado si no existe por parte de quien lo ofrece un estado de equilibrio de la salud física y mental. Sin embargo, momentos de dificultades y crisis relacionadas al trabajo pueden tener diferentes significados y formas $(3,4)$ y el uso de drogas psicoactivas generar un alivio de estas situaciones (5). Un estudio buscó identificar el uso de drogas psicoactivas, siendo los sujetos de estudio miembros del personal de enfermería de un hospital. La mayoría eran mujeres, casadas, católicas, de entre 45 a 61 años, con hijos, ingreso familiar de tres salarios mínimos, auxiliares de servicios médicos (asistentes), con 21 años de servicio, trabajando en sistemas de turnos de 12 x 36 h, desarrollando una segunda jornada 
laboral en su casa, sin espacios para el ocio, pero con sentimientos positivos en relación al trabajo. Sin embargo, como en otros estudios relativos a los trabajadores de enfermería $(3-6,8,16)$, el medio ambiente de trabajo era considerado estresante (7). Los sujetos informaron tener conocimiento de los efectos de las sustancias psicoactivas y consideraron que los problemas de salud mental son factores claves para el uso de estas sustancias, aun así el 48,5\% las había utilizado o todavía las utilizan, prevaleciendo los ansiolíticos en su mayoría prescritos a través de una receta médica del hospital donde trabajaban (25).

Otros autores realizaron entrevistas a trabajadores de enfermería de un hospital, con el objetivo de comprender cómo era para ellos hacer frente a las sustancias psicoactivas. Parecía ser un procedimiento como cualquier otro, sin embargo tuvieron desconfianza para hablar de ello. A medida que verbalizaban sus vivencias relacionadas con las drogas, los sujetos reconocieron la importancia de reflexionar y discutir el tema, y se mostraban poco preparados para hacer frente a situaciones que involucran el uso de estas sustancias. Se hicieron sugerencias para reconsiderar su formación profesional, teniendo en cuenta los problemas causados por el consumo excesivo, se sugirió que en el plan de estudios se podría incluir un debate sobre el tema, motivando a los futuros profesionales a darse cuenta de este fenómeno y de sus consecuencias (5).

En un estudio se analiza el uso y abuso de drogas psicoactivas por parte de personal de enfermería en relación con su salud. Se observó que el uso surgió como un elemento facilitador para hacer frente a las horas de trabajo, especialmente de noche, sin embargo, también causó problemas de salud de las enfermeras e interferencia en su trabajo. Se evidenció la necesidad de una mayor atención a la salud y educación permanente en materia de drogas, destinadas a ampliar la capacidad de hacer frente a este problema en los mismos trabajadores (26). El papel de los profesionales de enfermería en relación con el fenómeno del uso y abuso de las drogas es principalmente el de un educador (2).

Una investigación desarrollada con docentes de enfermería de una institución de educación superior ha estimulado la reflexión y el debate a nivel de pregrado, el contenido y las estrategias que pueden ayudar a los profesores y estudiantes para hacer frente al fenómeno de las drogas. Los resultados indicaron dificultades en los conocimientos, las estrategias, el diálogo y la relación de los profesores con los estudiantes en el abordaje de este problema, se evidenció la necesidad de preparar a los docentes para actuar en este contexto, ya que la inseguridad respecto al tema estuvo presente en su discurso. Hay deficiencias en la enseñanza de contenidos y también la forma de abordar el tema en el aula. La creación colectiva de la educación sobre drogas en el pregrado de enfermería se encuentra en progreso y se constituye en un desafío a ser enfrentado por todos (27). Los profesionales de enfermería son susceptibles a la dependencia de ciertas drogas, en particular las lícitas, por las posibilidades de mayor acceso a ellas en su lugar de trabajo (3, 4) y a la autoadministración (17).

Las drogas son utilizadas para intentar minimizar o revertir el síndrome de desgaste profesional, sin embargo con esto se quebrantan principios éticos y estéticos de la profesión (17) ya que el efecto de estas sustancias altera el comportamiento, el pensamiento lógico (3), la toma de decisiones y la ejecución de procedimientos especializados, poniendo en peligro la vida de las personas que reciben los cuidados de enfermería y la propia salud de los trabajadores (17). Resultados de un estudio en trabajadores de enfermería de un hospital muestran que los sujetos entendieron el uso de las drogas lícitas como algo natural y aceptable en el ámbito social. Se observó que el considerar el consumo de drogas como una solución para aliviar los problemas del ámbito familiar y del trabajo demuestra una trivialización del uso, 
porque sus efectos son perjudiciales para la salud, familia, trabajo y sociedad (4).

\section{CONSIDERACIONES FINALES}

El uso de drogas por parte del personal de enfermería es una realidad que debe ser abordada y resuelta. Puede estar asociada, entre varias razones: a la necesidad de aliviar las tensiones que se producen en el trabajo diario; para evitar o dejar de pensar en los problemas; las exigencias laborales; por la facilidad de acceso a estas sustancias; por inadecuadas condiciones ambientales, y dificultades familiares y sociales; por conflictos dentro y fuera del lugar de trabajo; el conocimiento incipiente sobre el tema; la falta de madurez personal; la influencia de colegas y amigos; para reducir al mínimo los síntomas que se presentan en el síndrome de desgaste profesional, entre otras razones.

Otro tema importante que emerge en el caso de los profesionales de enfermería y su formación académica es la malla curricular que, muchas veces, no ofrece en su pregrado asignaturas de salud de los trabajadores y necesariamente se requiere mostrar los riesgos a los cuales se enfrentará en su vida profesional. El estudiante tiene derecho a conocer cuáles son las condiciones laborales que implica el trabajo de enfermería, en la mayoría de los casos un mundo de trabajo nada seguro y saludable, sino por el contrario, los ambientes presentan problemas, las relaciones profesionales son a menudo difíciles, los salarios no son estimulantes, el contacto con los clientes y las familias pueden generar tensiones. No es fácil hacer frente a situaciones de estrés y sufrimiento que causan diariamente este conjunto de problemas, además de los de índole personal que pueden favorecer la ocurrencia de problemas a la salud cuando se convierta en un profesional.

Una forma de prevenir este problema es la enseñanza obligatoria de esta materia en los cursos de licenciatura en enfermería. Probablemente, entonces, con un mejor conocimiento del tema desde el pregrado, haya oportunidad de adelantar el reconocimiento de la dependencia a estas drogas y motivar la búsqueda de tratamiento. Así también se debería investigar más la información sobre las causas y consecuencias del uso y abuso de drogas en los diferentes contextos donde se desarrolla el quehacer de enfermería, como factores protectores que pueden frenar la motivación de un primer contacto o uso inicial.

Por otro lado, las instituciones empleadoras deberían ofrecer condiciones adecuadas de trabajo para el personal de enfermería para que éste desarrolle sus actividades con calidad. Deben saber también identificar cuando el problema del consumo de drogas se produce entre los trabajadores y promover la orientación y la derivación a un tratamiento adecuado, así como promover campañas de información para la prevención del uso indebido de drogas entre sus trabajadores, tratando de involucrar a todos los sectores de la sociedad en la prevención.

Las campañas preventivas pueden ser reunidas en una forma más integradora, mostrando todo tipo de drogas y sus formas de consumo, ya que, muchas veces, cada adicción tiene una conducta y un tratamiento específico y diferenciado. Equipos y clínicas de salud, en general, deberían facilitar a los usuarios de drogas la búsqueda de apoyo en los centros especializados para el tratamiento y la rehabilitación psicosocial y los miembros de la familia deben fomentar este proceso, que es, a menudo, el principal medio de apoyo emocional para un progreso de retorno a la salud.

Se reconoce que los estudios sobre este asunto complejo son necesarios con el fin de conocerlo mejor y con mayor profundidad, con el objetivo de minimizarlo y/o remediarlo. 


\section{REFERENCIAS}

1. Beck LM, David HMSL. O abuso de drogas e o mundo do trabalho: possibilidades de atuação para o enfermeiro. Esc. Anna Nery Rev Enferm. 2007; 11(4): 706-11.

2. Mendes IAC, Luis MAV. Uso de substâncias psicoativas, um novo velho desafio. Rev Lat Am Enfermagem. 2004; 12(spe): 295-300.

3. Martin ERC, Zeitoune RCG. As condições de trabalho como fator desencadeador do uso de substâncias psicoativas pelos trabalhadores de enfermagem. Esc. Anna Nery Rev Enferm. 2007; 11(4): 639-44.

4. Martins ERC, Zeitoune RCG, Francisco MTR, Spindola T, Marta CB. Concepções do trabalhador de enfermagem sobre drogas: a visibilidade dos riscos. R Enferm UERJ. 2009; 17(3): 368-72.

5. Martins ERC, Corrêa AK. Lidar com substâncias psicoativas: o significado para o trabalhador de enfermagem. Rev Lat Am Enfermagem. 2004; 12(spe): 398-405.

6. Stacciarini JM, Tróccoli B. O estresse na atividade ocupacional do enfermeiro. Rev Lat Am Enfermagem. 2001; 9(2): 17-25.

7. Elias MA, Navarro VL. A relação entre o trabalho, a saúde e as condições de vida: negatividade e positividade no trabalho das profissionais de enfermagem de um hospital escola. Rev Lat Am Enfermagem. 2006; 14 (4): 517-25.

8. Mauro MYC, Guimarães RM, Mauro CCC. Riscos ocupacionais em saúde. $\mathrm{R}$ Enferm UERJ [Internet]. 2004 [citado 4 abril 2010]; 12(3): 338-45. Disponible en: http://www.facenf.uerj.br/v12n3/ v12n3a14.pdf

9. Mauro MYC, Veiga AR. Problemas de saúde e riscos ocupacionais: percepções dos trabalhadores de enfermagem de unidade materna infantil. R Enferm UERJ [Internet]. 2008 [citado 4 abril 2010]; 16(1): 64-9. Disponible en: http://www.facenf. uerj.br/v16n1/v16n1a10.pdf

10. Nogueira-Martins LA. Saúde mental dos profissionais de saúde. Rev Bras Med Trab. 2003; 1 (1): 56-68.

11. Canini SRMS, Moraes AS, Gir E, Freitas ICM. Percutaneous injuries correlate in the nursing team of a Brazilian tertiarycare university hospital. Rev Lat Am Enfermagem. 2008; 16(5): 818-23.

12. Moreira AMR, Mendes R. Factores de riesgo de los disturbios óseos y musculares relacionados la labor de enfermería. $\mathrm{R}$ Enferm UERJ [Internet]. 2005 [citado 4 abril 2010]; 13(1): 19-26. Disponible en:http://www.facenf.uerj.br/v13n 1/ v13n1a03.pdf

13. Alexandre N. Aspectos ergonômicos relacionados com o ambiente e equipamentos hospitalares. Rev Lat Am Enfermagem. 1998; 6(4): 103-109.

14. Barboza D, Soler Z. Afastamentos do trabalho na enfermagem: ocorrências com trabalhadores de um hospital de ensino. Rev Lat Am Enfermagem. 2003; 11(2): 177-183.

15. Costa ES, Morita I, Martínez MAR. Percepção dos efeitos do trabalho em turnos sobre a saúde e a vida social em funcionários da enfermagem em um hospital universitário do Estado de São Paulo. Cad Saude Publica. 2000; 16(2): 553-55.

16. Martins JT. Prazer e sofrimento no trabalho do enfermeiro em unidades de terapia intensiva: estratégias defensivas [Tesis de Doctorado]. [Ribeirão Preto (SP)]: Escuela de Enfermería de Ribeirão Preto/USP; 2008.

17. Zeferino MT, Santos VEP, Radunz V, Carraro TE, Frello AT. Enfermeiros e uso abusivo de drogas: comprometendo o cuidado de si e do outro. R Enferm UERJ. [Internet]. 2006 [citado 4 abril 2010]; 14(4): 599-605. Disponible en: http:// www.facenf.uerj.br/v14n4/v14n4a17.pdf

18. Oliveira EB, Furegato ARF. Nursing students' work, a risk factor for the con- 
sumption of alcohol and other drugs. Rev Lat Am Enfermagem. 2008; 16(spe): 565-571.

19. Martis JT, Robazzi ML, Garanhani M. Sentimentos de prazer entre enfermeiros de unidades de terapia intensiva. Cienc enferm. 2009; 15 (30): 45-53.

20. Instituto de Medicina Social e de Criminologia de São Paulo, IMESC [Internet] INFO drogas. Como identificar um usuário. São Paulo: Instituto de Medicina Social e de Criminologia de São Paulo. [citado 19 julio 2008]. Disponible en: http://www.imesc.sp.gov.br/infodrogas/ usu\%C3\%A1rio.htm.

21. Ribeiro TCL, relator. O significado das substâncias psicoativas para o graduando de enfermagem. Poster presentado en: $10^{\circ}$ Congresso Brasileiro dos Conselhos de Enfermagem; 2007 Sept 3-7; Curitiba/ PR; Brasil.

22. Gabriel A, Tristão C, Izar L, Pina S, Franzin L, Ribeiro D et al. Consumo de Álcool e Drogas Ilícitas entre Estudantes de Medicina, Biologia e Enfermagem. Rev Fac Ciênc Méd Sorocaba. 2004; 6: 30-7.

23. Carvalho MTC. Convivência das jovens estudantes de enfermagem com o uso e abuso de drogas no contexto universitário [Tesis de Doctorado]. [Rio de Janeiro (RJ)]: Universidad Federal de Río de Janeiro; 2001.

24. Flores E, Iblin E, Luis MAV. Uso y actitudes relacionados a las drogas en las estudiantes de enfermería de la Universidad Mayor de San Andrés. Rev Lat Am Enfermagem. 2004; 12(spe): 376-82.

25. Pin JG. O profissional de enfermagem e a dependência química por psicofármacos: uma questão na saúde do trabalhador [Tesis de Mestría]. [Rio de Janeiro (RJ)]: Universidad Federal de Río de Janeiro; 1999.

26. Costa MS. O enfermeiro e as drogas: percepções a partir de um discurso coletivo [Tesis de Mestría]. [Rio de Janeiro (RJ)]: Universidad del Estado de Río de Janeiro; 2005.

27. Gonçalves SEF. O ensaio do tema drogas na formação do enfermeiro: uma construção coletiva [Tesis de Mestría]. [Rio de Janeiro (RJ)]: Universidad del Estado de Río de Janeiro; 2006. 